University of Nebraska - Lincoln

DigitalCommons@University of Nebraska - Lincoln

2010

Picosecond electron diffraction from molecules aligned by dissociation

\author{
Martin Centurion \\ University of Nebraska - Lincoln, martin.centurion@unl.edu \\ Peter Reckenthaeler \\ Max-Planck-Institut für Quantenoptik \\ Ferenc Krausz \\ Max-Planck-Institut für Quantenoptik \\ Ernst E. Fill \\ Max-Planck-Institut für Quantenoptik
}

Follow this and additional works at: https://digitalcommons.unl.edu/physicscenturion

Part of the Physics Commons

Centurion, Martin; Reckenthaeler, Peter; Krausz, Ferenc; and Fill, Ernst E., "Picosecond electron diffraction from molecules aligned by dissociation" (2010). Martin Centurion Publications. 17.

https://digitalcommons.unl.edu/physicscenturion/17

This Article is brought to you for free and open access by the Research Papers in Physics and Astronomy at DigitalCommons@University of Nebraska - Lincoln. It has been accepted for inclusion in Martin Centurion Publications by an authorized administrator of DigitalCommons@University of Nebraska - Lincoln. 


\title{
Picosecond electron diffraction from molecules aligned by dissociation
}

\author{
M. Centurion, P. Reckenthaeler, F. Krausz, and E. Fill \\ Max-Planck-Institut für Quantenoptik, Hans-Kopfermann-Str. 1, D-85748 Garching, Germany \\ Corresponding author - M. Centurion; present address: Department of Physics and Astronomy, \\ University of Nebraska-Lincoln, Lincoln, NE 68588-0111, USA; email mcenturion2@unl.edu
}

\begin{abstract}
In gas electron diffraction an averaging over statistical directions of the molecules takes place. This results in diffraction patterns in the form of isotropic rings which yield information only on the radial distribution function displaying the inter-atomic distances. We demonstrate that by dissociating molecules with linearly polarized light the pattern becomes anisotropic. In the experiments the iodide $\mathrm{C}_{2} \mathrm{~F}_{4} \mathrm{I}_{2}$ is dissociated and molecular difference intensities and difference radial distribution curves are measured for directions parallel and perpendicular to the direction of polarization. With picosecond temporal resolution the curves clearly demonstrate transient anisotropy and its decay by molecular rotation. This experiment is a first step towards the determination of structure and of ultrafast structural changes by electron diffraction from aligned molecules.
\end{abstract}

Keywords: electron diffraction, molecular structure, dissociation, alignment

\section{Introduction}

Electron diffraction is one of the key methods to determine the structure of molecules [1]. Combined with temporal resolution it can be used to investigate changes in molecular conformation [2]. Unfortunately, however, the statistical molecular directions in the gas phase result in an averaging of the patterns of individual molecules over the different directions. Standard evaluation procedures still allow determining the structure of small molecules by combining the information available, i.e. the radial distribution function with theoretical modeling.

This procedure becomes exceedingly complicated for larger molecules, since in this case radial distribution functions contain many overlapping inter-atomic distances. Combined with other methods - e.g. theoretical ab initio studies - it is still possible in many cases to obtain accurate structural information (see e.g. [3,4]).

Another possible way out of this dilemma is to align the molecules in the gas phase. A number of different alignment methods have so far been demonstrated (see [5], and references therein). They can be roughly classified into adiabatic and non-adiabatic ones. Non-adiabatic methods are to be preferred since they allow reconstructing the molecule in a fieldfree environment [6,7].

Electron diffraction from adiabatically aligned molecules has been demonstrated by Hoshina et al. [8,9]. In their experiment CS2 molecules were aligned in an intense nanosecond laser field. In the present study we align molecules in a fieldfree environment by taking advantage of the fact that the cross- section for dissociation of a molecule is proportional to the squared cosine of the angle between polarization and the direction of the dipole of the dissociative transition. In this way "selective alignment" of the undissociated molecules is achieved. This method, though not resulting in a high degree of alignment has the advantage that it is relatively straightforward and that the alignment occurs instantaneously after dissociation. We use 1,2-diiodotetrafuoroethane (sum formula $\mathrm{C}_{2} \mathrm{~F}_{4} \mathrm{I}_{2}$ ) for demonstrating the anisotropy of a diffraction pattern.

\section{Experimental setup}

The experimental geometry is sketched in Figure 1a. The molecules are introduced into the vacuum chamber using a gas jet and dissociated using a laser pulse. The reaction is then probed using an electron pulse which propagates in a direction orthogonal to both the gas jet and laser pulses. The diffraction pattern is recorded on a $2 \mathrm{D}$ detector, which includes a beam stop to block the directly transmitted electrons. The molecules are dissociated using a linearly polarized UV laser pulse. In order to generate anisotropic diffraction patterns, the laser polarization is set perpendicular to the propagation of the electron pulses (horizontal when projected to the detector in Figure 1a).

Figure $1 \mathrm{~b}$ shows the experimental setup in more detail. Each laser pulse is split in two parts, a trigger for the photoemission and a pump for exciting the molecules. The laser generates pulses with a duration of $50 \mathrm{fs}$, a central wavelength of $800 \mathrm{~nm}$ and maximum energy of $2 \mathrm{~mJ}$ at a repetition rate 

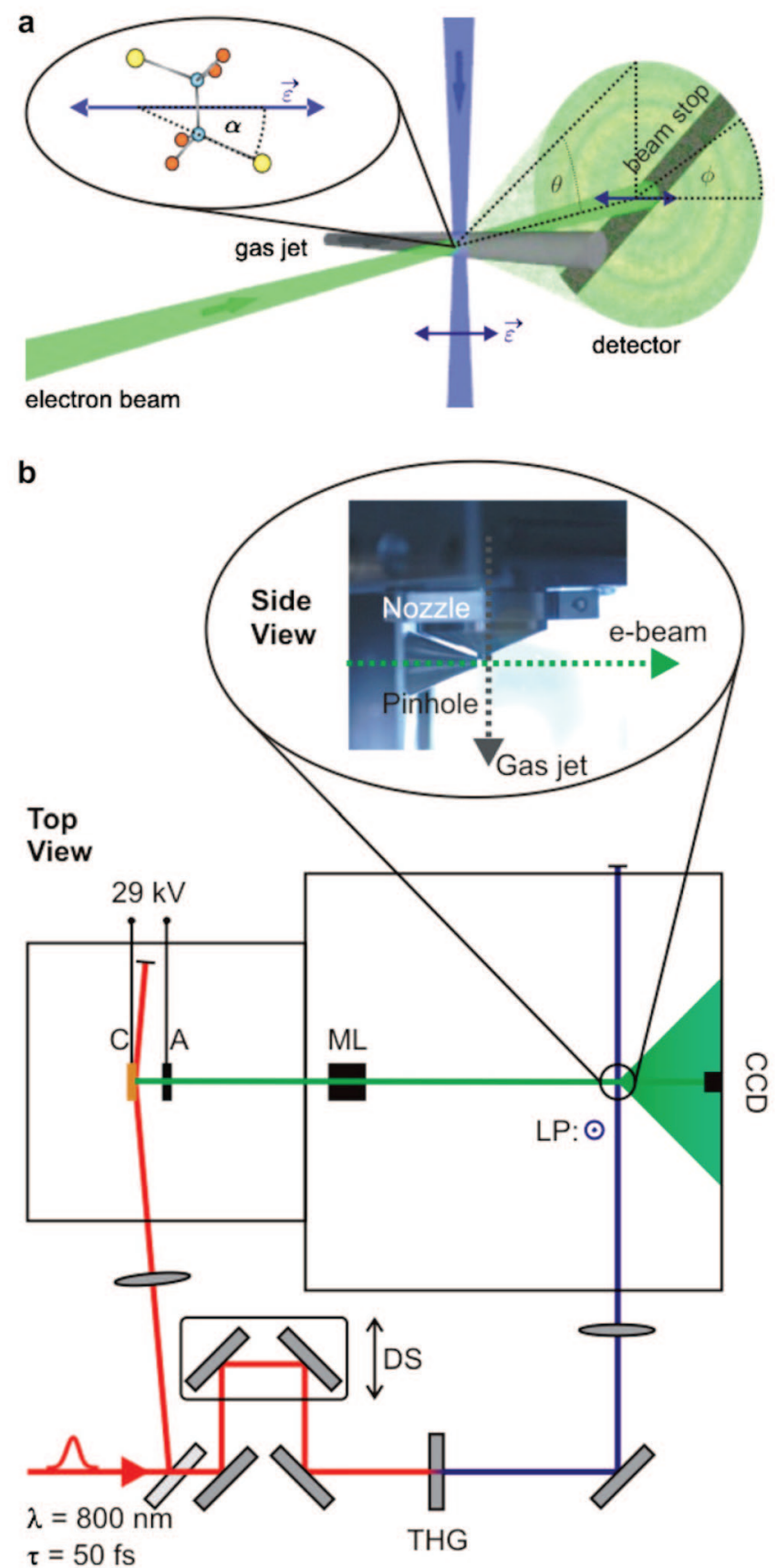

Figure 1. (a) Experimental geometry. Gas jet, electron beam and laser beam are mutually orthogonal. The $2 \mathrm{D}$ diffraction pattern is recorded. (b) Experimental setup: $\mathrm{C}=$ cathode, $\mathrm{A}=$ Anode, $\mathrm{ML}=$ Magnetic Lens, $\mathrm{DS}=$ delay stage, $\mathrm{THG}=$ third harmonic generation, $\mathrm{LP}=$ laser polarization.

of $1 \mathrm{kHz}$. A small fraction of the pulse energy is focused onto a copper photocathode to create a pulse of electrons by photoemission. Electrons are created through 3-photon absorption (the photon energy of $1.55 \mathrm{eV}$ is below the work function for copper). The photo-emitted electrons are accelerated by a static field to an energy of $29 \mathrm{keV}$ over a distance of $11 \mathrm{~mm}$. Two pairs of parallel plates with static electric fields are used to steer the electron beam, and a solenoid magnetic lens is used to focus it. The distance between the photocathode and the gas jet is 250 $\mathrm{mm}$. The electron beam is aligned onto the gas jet by the use of a pinhole $(200 \mu \mathrm{m}$ diameter) that is placed $1 \mathrm{~mm}$ before the gas jet (see inset in Figure 1b). The beam is focused such that a small spot is generated on the detector $(250 \mu \mathrm{m}$ diameter) while still achieving a good transmission of about 30\% through the pinhole. The detector consists of a phosphor screen that is fibercoupled to a cooled CCD with 16-bit dynamic range. The distance between gas jet and phosphor screen is $60 \mathrm{~mm}$.

The initial electron pulse duration follows that of the laser, but the electron pulses broaden as they propagate to the target. Their duration depends on the initial energy spread of the photoelectrons and on the space charge effect (Coulomb force between electrons). We used a Faraday cup to calibrate the number of electrons per pulse, which was approximately 12,000 in our experiments. Given our experimental conditions, the pulse duration is limited by space charge effects. We have calculated an electron pulse duration of 2.3 ps at the target using the General Particle Tracer (GPT) code [10]. The temporal resolution is further deteriorated by the velocity mismatch between photons and electrons as they traverse the target, which leads to a calculated effective resolution of about 3 ps. A laser wavelength in the UV region is necessary to dissociate the molecules. For this reason, the pump laser pulses are frequency tripled to $267 \mathrm{~nm}$ using two $1 \mathrm{~mm}$ BBO crystals. To optimize the conversion efficiency, the laser pulses are stretched to 100-150 fs before the crystals. UV-pulses with an energy of $100 \mu \mathrm{J}$ are focused to a diameter of $200 \mu \mathrm{m}$ in the gas jet. All three beams are approximately $200 \mu \mathrm{m}$ in diameter at the interaction region.

A seeded gas jet was used to reduce the rotational temperature of the molecules, thus increasing the time during which they remain aligned. First, Helium and $\mathrm{C}_{2} \mathrm{~F}_{4} \mathrm{I}_{2}$ are mixed at pressures of $270 \mathrm{mbar}$ and $30 \mathrm{mbar}$, respectively. A gas jet is then introduced into the experimental chamber through a de Laval nozzle which has an inner opening of $50 \mu \mathrm{m}$ diameter and outer opening of $150 \mu \mathrm{m}$. The backing pressure behind the nozzle varies between 227 mbar and 143 mbar for each experimental run.

Images are recorded both for the diffraction pattern and background for each time step. Each time step consists of 1216 images with an integration time of 5 min each. Background images are recorded interspaced with the diffraction images. The background is taken under the same conditions as the diffraction image but without the gas jet. The background is then subtracted from each diffraction image. Unusable pixels are removed from each image, i.e. the area behind the beam stop, the area outside of the fiber taper connected to the chip and pixels with very high absolute value (pixels that are defective or are hit by background radiation). The $2 \mathrm{D}$ image shown in Figure 2a is generated by combining the two halves separated by the diagonal behind the beam stop. The resulting images are denoised using a Wiener filter. The 2D patterns are converted to $1 \mathrm{D}$ curves (Figure $2 \mathrm{~b}$ ) by azimuthally averaging, either the whole image or using a limited range of angles.

\section{Theory}

The information on molecular structure is contained in the molecular scattering intensity $I_{\text {mol}}$ given by Schäfer [1].

$$
I_{\text {mol }}(s)=C \sum_{i=1}^{N} \sum_{k=1}^{N}\left|f_{i}\right|\left|f_{k}\right| \exp \left(-1 / 2 l_{i k}^{2} s^{2}\right) \cos \left(\eta_{i}-\eta_{k}\right) F_{i} k(s)
$$

where $C$ is a proportionality constant, $f_{i, k}$ are the scattering amplitudes of atoms $i, k, l_{i k}$ is the mean amplitude of vibration of atom pair $i-k, \eta_{i, k}$ represent the phase shift suffered by the electron wave, $s=4 \pi \sin (\Theta / 2) / \lambda$, where $\Theta$ is the scattering angle and $\lambda$ is the electron wavelength. $F_{i k}$ is the geometry factor to be discussed below. In the second sum of Equation (1) the case $k=i$ is excluded.

In the standard case of gas electron diffraction Imol is cylindrically symmetric and the factor Fik depends only on $s$ :

$$
F_{i k}=\frac{\sin \left(s r_{i k}\right)}{s r_{i k}}
$$




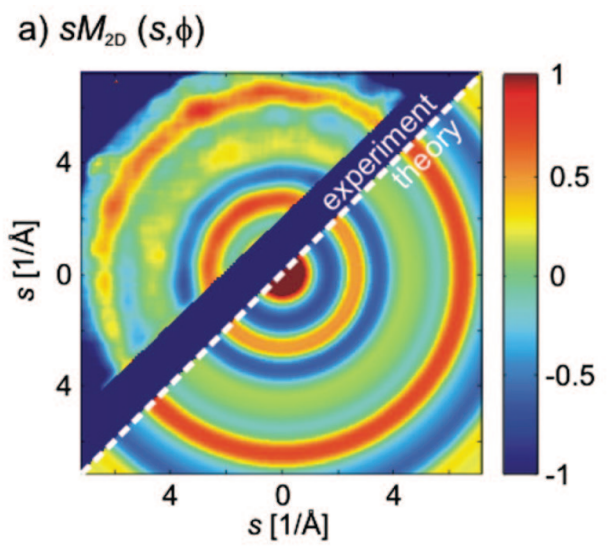

b) $s M(s)$

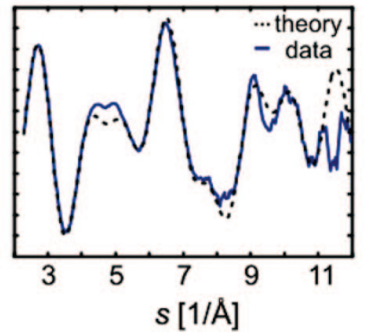

c) $f(r)$

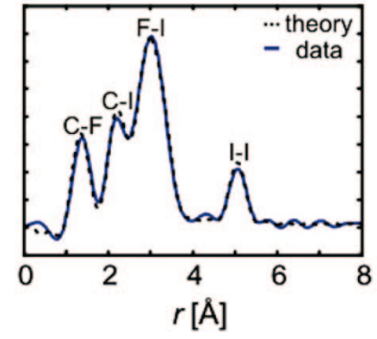

Figure 2. (a) Experimental and theoretical electron diffraction pattern $s M$ for $\mathrm{C}_{2} \mathrm{~F}_{4} \mathrm{I}_{2}$. (b) Azimuthally averaged $s M$. (c) Radial distribution function $f(r)$.

To evaluate the pattern the so called modified molecular scattering intensity $s M(s)$ is calculated by dividing the molecular intensity by the atomic intensity $I_{\text {at }}$ and multiplying by $s$, thus

$$
s M(s)=s \frac{I_{\mathrm{mol}}(s)}{I_{\mathrm{at}}(s)}
$$

In the case of isotropic gas electron diffraction the $s M(s)$ function is azimuthally averaged and subjected to a sine Fourier transform, yielding the radial distribution function $f(r)$ as

$$
f(r)=\int_{s_{\min }}^{s_{\max }} s M(s) \exp \left(-k_{d} s^{2}\right) \sin (s r) d s
$$

In this equation $s_{\min }$ and $s_{\max }$ are the minimum and maximum values of $s$ available from the experiment and $k_{d}$ is a damping factor introduced to suppress spurious high frequency oscillations which would occur at a hard cutoff. As described later, in our experiment $s_{\min }=2 \AA^{-1}$ and the theoretical $s M(s)$ values are used for the range $0<s<s_{\min }$.

In time-resolved electron diffraction the changes induced by the reaction are evaluated by calculating difference parameters $\Delta[s M(s)]$ between the diffraction pattern of the excited molecules and that of the ground state.

$$
\Delta[s M(s, t)]=s M(s, t)-s M(s, t<0)
$$

for which the $s M$ curve of the ground state is subtracted. Using the same procedure as in static diffractometry the radial distribution function is calculated yielding radial difference distribution functions $\Delta f(r)$.

In the case of an aligned molecular sample the diffraction pattern is not cylindrically symmetric but shows anisotropy. The case of alignment by selection through photodissociation has been treated by Williamson and Zewail [12] and Spencer Baskin and Zewail [11]. In these papers it was shown that in this case the geometrical factor occurring in Equation (1) is given by

$$
\begin{aligned}
F_{i k}(s, \Phi)= & \frac{j_{1}\left(s r_{i k}\right)}{s r_{i k}}-\left[\sin ^{2}\left(\Omega_{i k}\right)+\left(2-3 \sin ^{2}\left(\Omega_{i k}\right)\right) \cos ^{2}\left(\frac{\Theta}{2}\right) \cos ^{2}(\Phi)\right] \\
& \times \frac{j_{2}\left(s r_{i k}\right)}{2}
\end{aligned}
$$

In this equation the $j_{l}$ are the spherical Bessel functions of or$\operatorname{der} l$ and $\Omega_{i k}$ is the angle between $r_{i k}$ and the laser polarization.

Equation (6) shows that now the diffraction pattern depends on the azimuthal angle $\Phi$ and, moreover, that the angles of the interatomic vectors enter into the expression.

\section{Experimental results}

\subsection{Static diffraction}

Figure 2a shows the 2D scaled diffraction pattern $s M$ for $\mathrm{C}_{2} \mathrm{~F}_{4} \mathrm{I}_{2}$ before laser excitation, with good agreement between the experimental and calculated patterns. The molecule has two conformers, anti and gauche, both of which are dissociated. The theoretical pattern is calculated taking the two conformers at a ratio of 83/17 into account. This ratio is derived from the data in [13], taking our rotational temperature into account.

The experimental diffraction pattern is folded along the image diagonal. A diagonal beam stop is used because it will permit the measurement of the diffraction pattern in the directions parallel and perpendicular to the laser polarization. As expected, the diffraction pattern is isotropic due to the random orientation of the molecules in the gas phase. Figure $2 \mathrm{~b}$ shows the azimuthally averaged $s M$ curves.

Our data is in very good agreement with theory up to about $s=11 \AA^{-1}$, for higher scattering angles the signal is too weak and falls below the detection level of our apparatus. A higher s-range could be accessed by increasing the sensitivity of the detector. Figure $2 \mathrm{c}$ shows the radial distribution function $f(r)$ obtained from the curve in Figure 2b. Some of the inter-atomic distances contributing to the peaks are noted in the figure, see for example the peak at $5 \AA$, which corresponds to the I-I distance. Due to the random orientation of molecules in the gas phase, only 1D information about the molecule is accessible. As is clear from the reconstructed $f(r)$ function, only the inter-atomic distances are recovered, not the bond angles. As explained in the introduction this information is sufficient to fully reconstruct simple molecules, but is not enough for more complicated structures.

\subsection{Time-resolved diffraction}

In this section we show our results on electron diffraction from molecules aligned by photodissociation. The molecules are dissociated using a linearly polarized UV femtosecond laser pulse. The corresponding absorption cross-section is proportional to $\cos ^{2}(\alpha)$, where $\alpha$ is the angle between the laser polarization and the direction of the transition dipole moment (see Figure 1a). For $\mathrm{C}_{2} \mathrm{~F}_{4} \mathrm{I}_{2}$ the transition dipole moment of the most intense transition is parallel to the C-I bond, along which the dissociation takes place. Therefore, the $\mathrm{C}_{2} \mathrm{~F}_{4} \mathrm{I}$ radicals emerge preferentially with the dissociated $\mathrm{C}-\mathrm{I}$ direction aligned along the laser polarization vector. Due to the rotation of the molecules the alignment decays with time. The time constants of the reaction have been determined using mass spectrometry [14].

$$
\mathrm{C}_{2} \mathrm{~F}_{4} \mathrm{I}_{2} \rightarrow(200 \mathrm{fs}) \mathrm{C}_{2} \mathrm{~F}_{4} \mathrm{I}+\mathrm{I} \rightarrow(25 \mathrm{ps}) \mathrm{C}_{2} \mathrm{~F}_{4}+2 \mathrm{I}
$$

The first iodine atom breaks off within $200 \mathrm{fs}$, leading to the intermediate $\mathrm{C}_{2} \mathrm{~F}_{4} \mathrm{I}$ radical. Subsequently, approximately $30 \%$ of the radicals fragment (with a time constant of $25 \mathrm{ps}$ ) to yield $\mathrm{C}_{2} \mathrm{~F}_{4}$. In this paper we focus on the early stages of the reaction, where the anisotropy is present. The complete reaction has 
a) Parallel to laser polarization

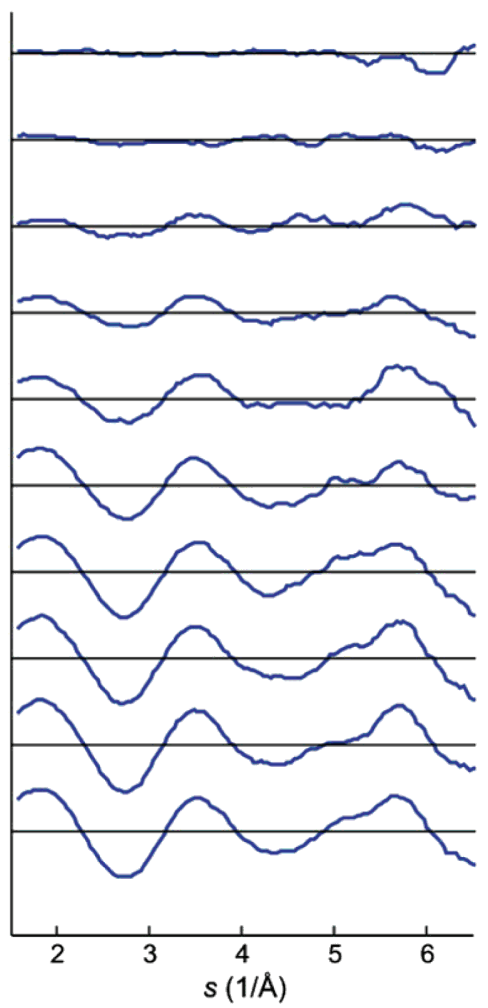

b) Perpendicular to laser polarization

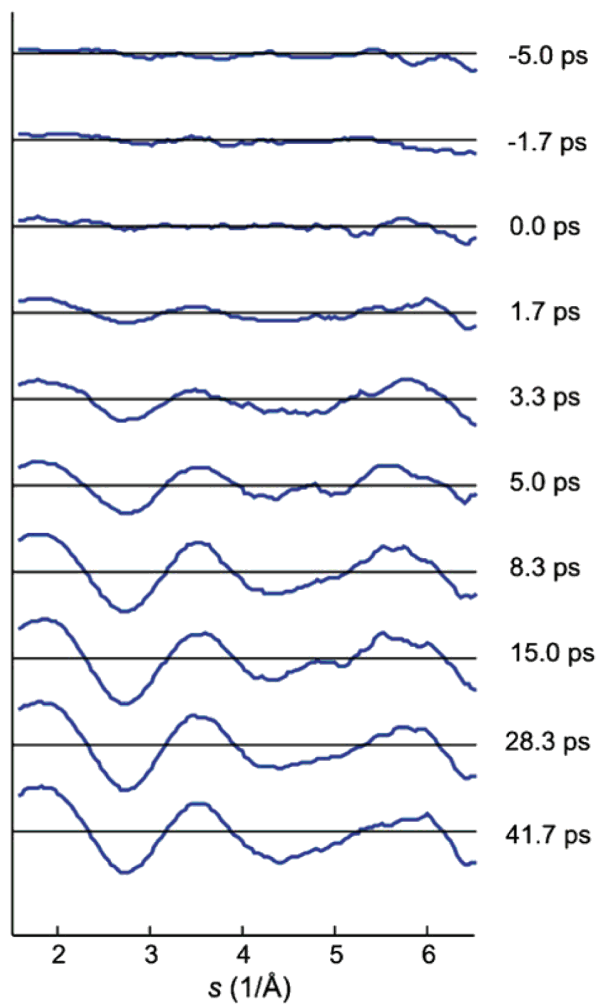

Figure 3. $\Delta s M$ curves for time delays between $-5.0 \mathrm{ps}$ and $41.7 \mathrm{ps}$. (a) Diffraction pattern in the angular region parallel to the laser polarization. (b) Diffraction pattern in the angular region perpendicular to the laser polarization.

been previously studied up to hundreds of picoseconds after the dissociation [15]. The structure of the intermediate $\mathrm{C}_{2} \mathrm{~F}_{4} \mathrm{I}$ has been determined using ultrafast electron diffraction [16].

In a previous publication [17] we showed the diffraction patterns at three time steps: immediately after dissociation when there is a strong anisotropy, a short time later when the anisotropy is weaker and at a later time when the anisotropy vanishes. Here we show the full temporal evolution of the difference molecular scattering DsM parallel and perpendicular to the laser polarization and also the corresponding difference radial distribution function $\Delta f(r)$.

The parallel molecular intensity is evaluated by averaging over a limited range of angles, using

$$
I_{\mathrm{mol},||}(s)=\frac{1}{2 \pi s} \int_{0}^{2 \pi} I_{\mathrm{mol}}(s, \Phi) \cos ^{12}(\Phi) d \Phi
$$

The perpendicular intensity $I_{\text {mol, } \perp}$ is calculated similarly, but replacing the cosine function in Equation (7) by a sine function. The $\cos ^{12}$ and $\sin ^{12}$ factors in the integral correspond to cones with an angular aperture of $38^{\circ}$. This value is a compromise to include sufficient data for a good signal to noise ratio, while maintaining a significant difference for the two orientations.

Our results show that the dissociation signal is observed immediately after dissociation only in the direction parallel to the laser polarization, and only at later times in the perpendicular direction. Furthermore, we now calculate also $\Delta f(r)$ in the two orthogonal directions (parallel and perpendicular to the laser) to demonstrate that different molecular structures are observed in different angular ranges.

Figure 3 shows the $\Delta s M$ curves for time steps between -5.0 ps and 41.7 ps. The curves display the difference in $s M$ before and after laser excitation (cf. Equation (5)). Negative times indicate that electrons arrive at the target before the laser. Figures $3 a$ and $3 \mathrm{~b}$ show the results corresponding to the directions parallel and perpendicular to the laser polarization, respectively. The lower limit in the s-range is determined by the beam stop used to prevent saturation due to the directly transmitted beam. The scattering cross-section decreases rapidly with $s$, so as expected the noise level increases with $s$. Our $\Delta s M$ curves show good signal to noise ratio up to about $s=6.5 \AA^{-1}$.

We can observe the evolution of the dissociation with high temporal resolution. We define the zero of time as the time when the first changes are observed. The curves before $t=0$ show the noise level of the data. We can discriminate the beginning of the dissociation process within a time step of $1.7 \mathrm{ps}$. It can be clearly seen that at $t=0 \mathrm{ps}$ a dissociation signal appears in the direction parallel to the laser, while nothing is observed in the perpendicular direction. This signal corresponds to the breaking of a C-I bond, as explained below. In both cases no changes are observed at $t=-1.7 \mathrm{ps}$. At $t=1.7 \mathrm{ps}$ the dissociation signal appears also in the perpendicular direction. In the following time steps up to 5 ps the signal continues to grow in both directions, with the signal in the parallel direction been markedly stronger. The increase in the signal over a few picoseconds is due to the convolution of the bond-breaking (200 fs) with the time response of our system (4 ps). However, the difference in the signals in the two directions is a clear indication that the molecules are not randomly oriented but partially aligned. In the time steps after 8.3 ps no significant difference is observed in the two directions, indicating that the orientation of the molecules has become again isotropic.

In Figure 4 we display the $\Delta f(\mathrm{r})$ corresponding to the $\Delta s M$ curves shown in Figure 3. The curves are calculated by filling in the missing values in the range of $0<s<2 \AA^{-1}$ with the theoretical values, and then performing the sine transform as de- 
a) Parallel to laser polarization

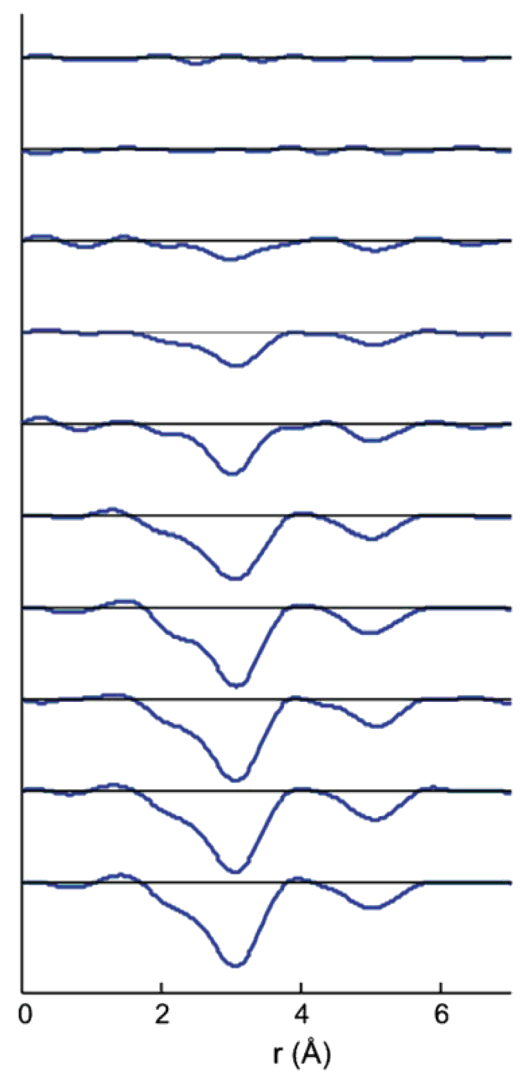

b) Perpendicular to laser polarization

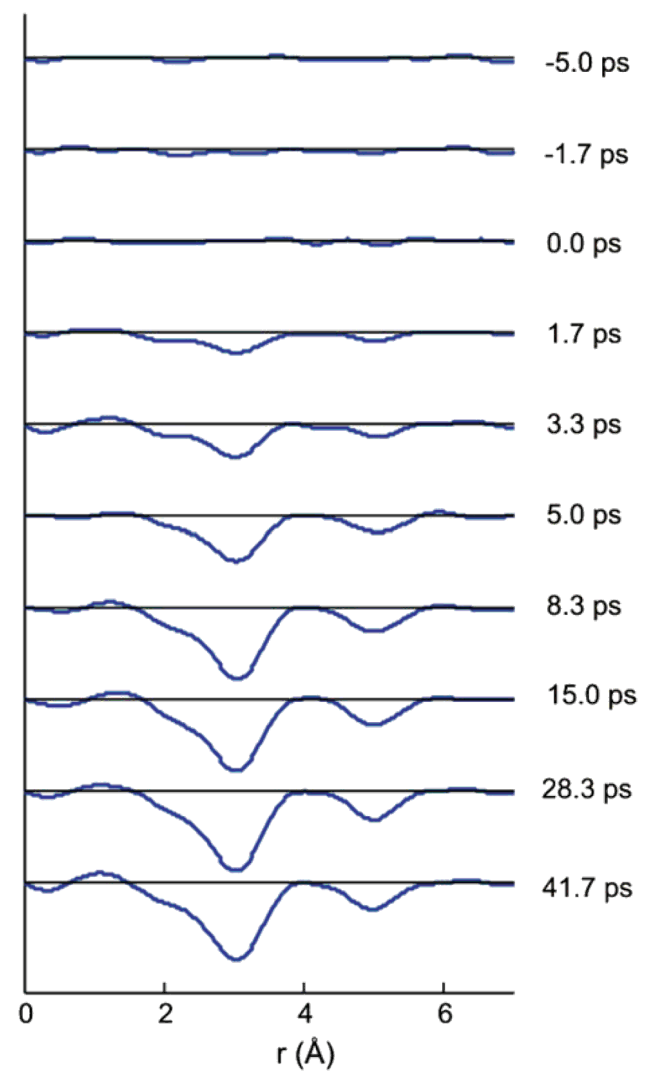

Figure 4. $\Delta f(r)$ curves for time delays between $-5.0 \mathrm{ps}$ and $41.7 \mathrm{ps}$. (a) Calculated from the $\Delta s M$ in Figure $3 \mathrm{a}$. (b) Calculated from the $\Delta s M$ in Figure $3 \mathrm{~b}$.

scribed in Section 3. As expected, no changes are observed before for $t<0 \mathrm{ps}$. At $t=0 \mathrm{ps}$ we see negative peaks appearing in the parallel direction, while no changes are visible in the perpendicular direction. The peaks are negative because they correspond to a depletion of these interatomic distances after dissociation. The peak at $5 \AA$ corresponds to the I-I atomic pair, while the peak at $3 \AA$ corresponds to the F-I pairs. The weaker peak due to the C-I pair is barely resolved at around $2 \AA$. Due to the limited s-range, our spatial resolution is limited to $\sim 1$ $\AA$. However, this is sufficient to conclude that at $t=0$ ps we detect the breakage of the $\mathrm{C}-\mathrm{I}$ bond only along the direction parallel to the laser polarization. Thus, we have succeeded in measuring different molecular structures in different angular ranges of the diffraction pattern. In the direction parallel to the laser polarization we detect a mixture of the ground state molecule $\mathrm{C}_{2} \mathrm{~F}_{4} \mathrm{I}_{2}$ and the radical $\mathrm{C}_{2} \mathrm{~F}_{4} \mathrm{I}$, while in the perpendicular direction we detect only the ground state (i.e. no changes in the diffraction pattern).

For later times the evolution is similar to that described before. After 1.7 ps the effect of dissociation can be seen also in the perpendicular direction, although with a weaker strength up to about 5 ps. For the time steps after 8.3 ps there is no significant difference in the two directions.

\section{Discussion and conclusion}

We interpret our result as follows: when the molecules are dissociated an anisotropic distribution is generated, i.e. the dissociated molecules are aligned preferentially along the direction of laser polarization. This angular distribution is captured at $t=0 \mathrm{ps}$ in our experiment. Immediately after dissociation the alignment starts to vanish as the molecules rotate. We expect the ground state molecules and the radicals to rotate with different time constants due to the different moment of inertia. However, due to the limited resolution of our experiment we are not able to distinguish between the angular distribution of the two different structures, and what we measure is an overall lifetime of the alignment of $2.6 \pm 1.2$ ps. This is consistent with the time constant of the ground state. Note that we dissociate at most $30 \%$ of the molecules.

Our results demonstrate for the first time the feasibility of recording temporally and angularly resolved diffraction patterns from aligned molecules. These results provide a first step towards the 3D reconstruction of transient molecular structures. While traditional electron diffraction provides 1D information on the inter-atomic distances $f(r)$, 3D information would allow studying larger and more complicated molecules. A projection of the 3D structure could be reconstructed froma single diffraction pattern by aligning molecules with a linearly polarized laser pulse [18], in which case the molecule is aligned along one axis. Alternatively, molecules could be aligned along two axes using elliptically polarized lasers, or a sequence of two laser pulses, and the structure reconstructed using suitable algorithms [19]. These alignment methods have been previously demonstrated in the case of 1D [6] and 3D alignment [20, 21].

\section{Acknowledgments}

This work was supported by DFG under Contract No. SFB Transregio 6039 and by the DFG Cluster of Excellence "Munic Centre for Advanced Photonics." P.R. is sponsored by the International Max-Planck-Research School on Advanced Photon Science-IMPRS-APS. M.C. was supported by the Alexander von Humboldt Foundation. 


\section{References}

[1] L. Schäfer, Appl. Spectrosc. 30 (1976) 123.

[2] J. R. Helliwell, P.M. Rentzepis, Time-Resolved Diffraction, Clarendon Press, Oxford, 1997.

[3] M. Dakkouri, H. Oberhammer, J. Mol. Struct. 102 (1983) 315.

[4] S. L. Hinchley, D. A. Wann, D.W.H. Rankin, Int. J. Quantum Chem. 101 (2005) 878.

[5] H. Stapelfeldt, T. Seideman, Rev. Mod. Phys. 75 (2003) 543.

[6] L. Holmegaard, J. H. Nielsen, I. Nevo, H. Stapelfeldt, F. Filsinger, J. Küpper, G. Meijer, Phys. Rev. Lett. 102 (2009) 023001.

[7] E. Peronne, M. D. Poulsen, C. Z. Bisgaard, H. Stapelfeldt, T. Seideman, Phys. Rev. Lett. 91 (2003) 043003.

[8] K. Hoshina, K. Yamanouchi, T. Ohshima, Y. Ose, H. Todokoro, Chem. Phys. Lett. 353 (2002) 27.

[9] K. Hoshina, K. Yamanouchi, T. Ohshima, Y. Ose, H. Todokoro, J. Chem. Phys. 118 (2003) 6211.

[10] http://www.pulsar.nl/gpt

[11] J. Spencer Baskin, A. H. Zewail, ChemPhysChem 6 (2005) 2261.
[12] J. C. Williamson, A. H. Zewail, J. Phys. Chem. 98 (1994) 2766.

[13] H. Thomassen, S. Samdal, K. Hedberg, J. Am. Chem. Soc. 114 (1992) 2810.

[14] D. Zhong, S. Ahmad, A. H. Zewail, J. Am. Chem. Soc. 119 (1997) 5978.

[15] R. Srinivasan, V. A. Lobastov, C.-Y. Ruan, A. H. Zewail, Helv. Chim. Acta 86 (2003) 1763.

[16] H. Ihee, V. A. Lobastov, U. M. Gomez, B. M. Goodson, R. Srinivasan, C.-Y. Ruan, A. H. Zewail, Science 291 (2001) 458.

[17] P. Reckenthaeler, M. Centurion, W. Fuß, F. Krausz, E. Fill, Phys. Rev. Lett. 102 (2009) 213001.

[18] D. K. Saldin, V. L. Shneerson, D. Starodub, J.C.H. Spence, Acta Cryst. A66 (2010) 32.

[19] J. R. Fienup, Appl. Opt. 21 (1982) 2758.

[20] J. J. Larsen, K. Hald, N. Bjerre, H. Stapelfeldt, T. Seideman, Phys. Rev. Lett. 85 (2000) 2470.

[21] K. F. Lee, D. M. Villeneuve, P. B. Corkum, A. Stolow, J. G. Underwood, Phys. Rev. Lett. 85 (2006) 173001 